\title{
Polysaccharide-Based Nanomaterials for Ocular Drug Delivery: A Perspective
}

\author{
Haozhe Yu ${ }^{1,2}$, Wenyu $\mathrm{Wu}^{2}$, Xiang Lin $^{3}$ and Yun Feng ${ }^{1,2 *}$ \\ ${ }^{1}$ Institute of Medical Technology, Peking University Health Science Center, Beijing, China, ${ }^{2}$ Department of Ophthalmology, \\ Peking University Third Hospital, Beijing, China, ${ }^{3}$ School of Chinese Medicine, The University of Hong Kong, Hong Kong, \\ China
}

Ocular drug delivery is one of the most challenging issues in ophthalmology because of the complex physiological structure of the eye. Polysaccharide-based nanomaterials have been extensively investigated in recent years as ideal carriers for enhancing the bioavailability of drugs in the ocular system because of their biocompatibility and drug solubilization. From this perspective, we discuss the structural instability of polysaccharides and its impact on the synthesis process; examine the potential for developing bioactive polysaccharide-based ocular drug nanocarriers; propose four

OPEN ACCESS

Edited by: Jiaxu Hong,

Fudan University, China

Reviewed by:

Jui-Yang Lai,

Chang Gung University, Taiwan

Rocktotpal Konwarh,

Addis Ababa Science and Technology University, Ethiopia

*Correspondence:

Yun Feng

fengyun@bjmu.edu.cn

Specialty section:

This article was submitted to

Nanobiotechnology,

a section of the journal Frontiers in Bioengineering and

Biotechnology

Received: 31 August 2020 Accepted: 05 November 2020 Published: 10 December 2020

Citation:

Y U H, Wu W, Lin X and Feng Y (2020) Polysaccharide-Based Nanomaterials for Ocular Drug

Delivery: A Perspective.

Front. Bioeng. Biotechnol. 8:601246.

doi: 10.3389/fbioe.2020.601246 strategies for designing novel drug delivery nanomaterials; and suggest reviewing the behavior of nanomaterials in ocular tissues.

Keywords: polysaccharide-based nanomaterials, polysaccharides, ocular drug delivery, nanocarriers, nano

\section{INTRODUCTION}

Given the emerging popularity of electronic devices and the corresponding changes in lifestyle, the incidence of ophthalmologic diseases has increased rapidly. It has been speculated that the number of blind individuals will add up to 702 million by 2050 (Flaxman et al., 2017). However, the efficacy of ocular drug delivery has remained challenging in clinical management, mainly because of the unique anatomy and complex physiological barriers of the eye. For anterior diseases, including dry eye syndrome, conjunctivitis, and keratitis, eye drops are recognized as common treatments because of their high accessibility at the ocular surface (Jian et al., 2017; Li et al., 2019). However, ocular surface barriers and associated stress responses, such as tearing, cause eye drops to have poor bioavailability, and hence frequent instilling is required to maintain an effective drug concentration (Bennett et al., 2020). The posterior of the eye mainly includes the vitreous body, choroid, and retina, which maintain distance from the ocular surface and barrier layers, such as the choroidal circulation region and the blood-retinal barrier. For the posterior, intravitreal injection has been adopted for direct drug delivery. Nonetheless, this method remains difficult for patients to accept due to possible side effects, such as endophthalmitis and trypanophobia and the associated economic burdens (Müller et al., 2017). The ideal ophthalmic drug delivery system should be simple and non-invasive with ensured bioavailability. In recent decades, increasing studies have reported nanoscale materials as novel ocular drug carriers considering their enhanced tissue permeation and sustained release characteristics (Lakhani et al., 2018; Lynch et al., 2019; Qamar et al., 2019).

Polysaccharides are polymer compounds consisting of many monosaccharide molecules connected by glycosidic bonds. They can be divided into homopolysaccharides (composed of single monosaccharides, such as starch and cellulose) and heteropolysaccharides (composed of different 
monosaccharides, i.e., mucopolysaccharide). The physical characteristics of polysaccharides suggest distinct structural and physicochemical features, including chemical composition, degree of polymerization, number of branches, and surface charge, which are advantageous for designing drug carrier systems with adjustable characteristics in terms of drug loading content, release capacity, and biodistribution (Bernd et al., 2004; Furlani et al., 2019; Jin et al., 2020; Soliman et al., 2020). Compared with synthetic nanocarriers, polysaccharide-based nanomaterials exhibit a better performance in terms of drug retention and ocular permeability via the interpenetration of mucin chains, both directly and indirectly (Servais et al., 2018; George and Suchithra, 2019; Pathak, 2019). For example, chitosan, a type of polysaccharide with a linear structure and positive charge, can closely integrate with the cornea and conjunctiva, which have a negative surface charge. Consequently, this electrostatic interaction prolongs the retention time and enhances the penetration capability (Irimia et al., 2018). Moreover, polysaccharides are commonly present in the eye, such as hyaluronic acid, one of the major components of the vitreous body. Emerging studies have suggested the biocompatibility of polysaccharides and their derivative nanomaterials for ocular delivery (Nishikawa and Tamai, 1996; Nakagawa et al., 1997; De Oliveira Fulgêncio et al., 2012; Lodhi et al., 2020). Increasing evidence indicates their biosafety, good tolerance, and superior bioavailability, and polysaccharide-based nanocarriers have received considerable attention for clinical practice (Dubashynskaya N. et al., 2020). This perspective focuses on the structural instability of polysaccharides in synthesizing nanocarriers and provides a glimpse into their translational potential, including bioactive polysaccharide-based nanomaterials, novel strategies in nanocarrier design, and their behavior in ocular therapy (Figure 1).

\section{STRUCTURAL INSTABILITY OF POLYSACCHARIDES}

Quality control is a critical challenge for translational studies of polysaccharide-based nanocarriers from bench to bedside. Control of the physical-chemical properties in large-scale nanocarrier synthesis has been fully discussed elsewhere (Nagarwal et al., 2010; Bazile, 2014; Felice et al., 2014). However, the structural instability of the parent polysaccharides is often overlooked. Polysaccharides are widespread in various natural sources, including animals, plants, microbes, and algae, and techniques for their isolation and purification are the foundation for developing polysaccharide-based nanomaterials (Mozammil Hasnain et al., 2019). Thus, polysaccharides are readily accessible compared with proteins and nucleic acids because the latter two can encounter conformational changes upon extraction. Nonetheless, emerging evidence suggests a sort of structural instability in polysaccharides during extraction. For example, an increase in the molecular weight of hemicellulose was observed in a high-temperature aqueous alkali environment. Compared with barium hydroxide, hemicellulose is more vulnerable to degradation when exposed to organic alkaline compounds (Bian et al., 2010, 2012). Moreover, the viscosity of chitosan extracted from fungi is lower than that of crustaceous chitosan (Żukiewicz-Sobczak et al., 2015). Recent studies have demonstrated that different sources and separation methods may result in variances in the purity, molecular weight, and substituents of hyaluronic acid and chondroitin sulfate, which subsequently affect their bioactivities (Abdallah et al., 2020). Chang et al. reported that chitosan extracted from Auricularia sp. elicited better antibacterial activities than commercial chitosan (Chang et al., 2019). These differences will most likely lead to unpredictable drug-release behaviors from the synthesized polysaccharide-based nanomaterials and thus cause unstable therapeutic effects via the ocular nanocarriers. Even for several commercialized polysaccharides, such as cellulose, chitosan, and hyaluronic acid, many structural properties, such as chain length, degree of polymerization, and polydispersity, can vary between batches, resulting in unreproducible experimental results. To deal with this instability, reports on nanomaterial preparation should include detailed structural characterization of the employed polysaccharides, whether extracted or commercialized. In this regard, given the functional importance of the physicochemical and biological properties of synthesized ocular nanocarriers, systematic review methods could promote classification algorithms for polysaccharide processes.

To scale up the production of polysaccharide-based nanocarriers, the structural instability must be overcome, particularly for polysaccharides with branched-chain or special functional groups like mucopolysaccharides. From this point of view, it seems difficult to standardize the industrial production of polysaccharides by pharmaceutical companies worldwide as well as specified polysaccharide derivatives without standard guidelines. Therefore, consensus and standards covering properties including chain length, polymolecularity, degree of substitution, and monosaccharide composition are a high priority.

\section{BIOACTIVE POLYSACCHARIDE-BASED NANOCARRIERS FOR OCULAR DELIVERY}

Existing studies on ocular nanocarriers have mainly focused on commercialized polysaccharides, including cellulose, chitosan, and hyaluronic acid, because of their their easy access and ready usage for nanocarrier synthesis without needing additional preparation. In contrast, little is known about the properties, modification reactions, and ophthalmological applications of polysaccharides extracted from natural sources. Many studies have suggested that certain natural polysaccharides, particularly those from plants, possess unique biological activities for the ocular system. Lycium barbarum polysaccharides have been reported to ameliorate dry eye disease, prevent oxidative damage in human trabecular meshwork cells, and preserve the function, ganglion cells, and pigment epithelium cells of the retina (Yang et al., 2017; Yang M. et al., 2020; Chien et al., 2018; Lakshmanan et al., 2019; Liu and Zhang, 2019; Liu L. et al., 2020). Carboxymethyl Terminalia catappa polysaccharides 


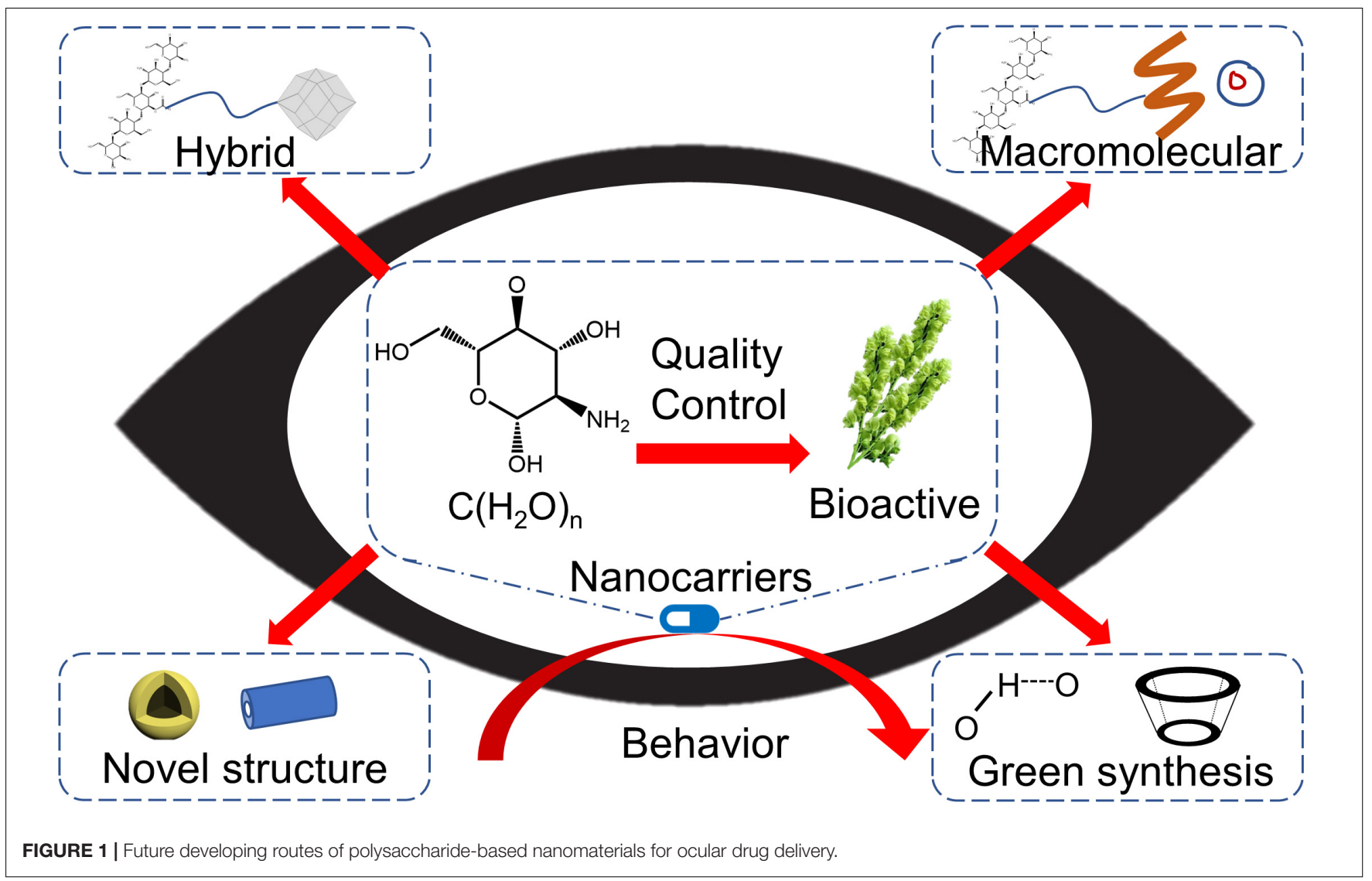

show therapeutic effects in the cornea from blue light-emitting diodes. Moreover, Polygonatum sibiricum polysaccharides could attenuate the damage from diabetic retinopathy, while dry eyes can be relieved by mucoadhesive Bletilla striata polysaccharidebased artificial tears (Chandel et al., 2019; Wang et al., 2019c; Thacker et al., 2020). Evidence has also demonstrated that phytobased polysaccharides can be utilized for drug delivery systems upon graft modification or synergistic interaction via noncovalent stabilization with other commercial polysaccharides such as hyaluronic acid (Uccello-Barretta et al., 2013; Wang et al., $2019 b)$. There is no doubt that leveraging this feature to develop dual-functional polysaccharide-based nanocarriers serving both carrier and therapeutic functions holds great potential from a clinical point of view.

The quality control of natural active polysaccharides has been challenging for developing ophthalmic drug-delivery systems and FDA approval. In addition to separation methods, it is also difficult to define certain polysaccharides with differential constituents, such as those from $P$. sibiricum, $P$. cyrtonema, $P$. kingianum, and $P$. odoratum; despite all being referred to as the so-called $P$. sibiricum polysaccharide, they present various saccharide mappings in high-performance gel permeation chromatography (Zhao et al., 2020). Moreover, emerging quality control methods for polysaccharides have been proposed, including polysaccharide receptor theory and multiple-fingerprint analysis (Cao et al., 2019; Li et al., 2020; Wang et al., 2020). In this context, the collaboration of experts in the fields of phytochemistry, analytical chemistry, and pharmacology is the highest priority to classify the structures of botanical polysaccharides for medical use.

\section{TYPICAL OCULAR POLYSACCHARIDE-BASED NANOCARRIERS AND NOVEL DESIGN STRATEGIES}

Polysaccharides show high chemical activities because of their abundant functional groups, such as amino and hydroxyl groups (Yang Y. et al., 2020). Several reviews have summarized the synthetic protocols for common polysaccharide derivatives (Tiwari and Bahadur, 2019; Anpilova et al., 2020; Yang Y. et al., 2020). In the nanomaterial synthesis process, polysaccharides can be reacted under drastic conditions, such as high temperatures, salt contents, and shear forces, with relatively stable structures and biological activities compared with nucleic acids and proteins (Yuan et al., 2018; Garcia-Vaquero et al., 2019; Wang et al., 2019a; Chao et al., 2020; Chen B. et al., 2020). Therefore, by using polysaccharides and their derivatives, nanomaterials with different types of geometric structures can be prepared, from zero- to three-dimensional, through various synthetic methods, such as hydrothermal synthesis and co-precipitation (Ferreira et al., 2020; Alizadeh-Sani et al., 2021; Pan et al., 2021). 
Nanoparticles, nanocapsules, and nanomicelles are typical zerodimensional drug carriers; nanoparticles are characterized by a uniform distribution of the drug and carrier, while the drug in the latter two is encapsulated in the carrier core. These nanomaterials improve the drug solubility and enhance the corneal penetration with prolonged retention times (Liu et al., 2019; Karava et al., 2020). One-dimensional polysaccharide-based nanomaterials are often used as excipients for their plasticity and low-encapsulation efficiency. For example, nanocellulose with its high mechanical strength can act as a reinforcing material (Taheri et al, 2020). Starting from one-dimensional nanofibers, the common two-dimensional fibrous membrane can be synthesized by electrospinning. Because of the high drug-loading capacity, long retention time, and sustained release of such membranes, they are often utilized as ocular inserts (Zheng et al., 2018). The properties of nanofibers dramatically affect the drug delivery performance (Mirzaeei et al., 2018; Yellanki et al., 2019). Nanogels are threedimensional hydrophilic networks on the nanometer scale that are capable of encapsulating drugs or biological macromolecules while maintaining their native conformation. One important feature is that certain nanogels with functional groups (e.g., disulfide moieties) are stimuli-responsive. Exogenous stimuli, including temperature, $\mathrm{pH}$, and ionic strength, can significantly influence the drug release kinetics and degradation rate; thus, nanogels are also called intelligent delivery systems (Chen S. et al., 2020; Lai et al., 2020).

Several strategies have been developed to maximize the drug delivery capacity of polysaccharide-based nanomaterials. A feasible design could be achieved using hybrid nanomaterials with polysaccharides. Polysaccharides can serve as stabilizers for other components, such as functional nanomaterials and biological macromolecules, in constructing polysaccharidebased nanohybrids (Zhan et al., 2019; Hammi et al., 2020). The participation of polysaccharides can also enhance the biocompatibility and biodegradability of exogenous materials, especially inorganic nanoparticles (Fan et al., 2019; Alaei et al., 2020; Dincă et al., 2020; Zheng et al., 2020). Santana et al. prepared $\mathrm{ZnS}$-coated CdS quantum dots and $\mathrm{ZnS}$ coated $\mathrm{AgInS}_{2}$ quantum dots by an aqueous route using chitosan conjugated with bevacizumab. Animal experiments showed no significant changes in electroretinography, intraocular pressure, histological, morphometric, or immunohistochemical examinations, indicating the good biocompatibility and biosafety of chitosan for ocular delivery (Santana et al., 2020). In addition, many studies have reported that the addition of precious-metal nanoparticles, such as silver, and carbon materials, such as graphene, can improve the antibacterial activity and release capacity (Shi et al., 2016; Joz Majidi et al., 2019; Asghar et al., 2020; Shah et al., 2020; Sun et al., 2020).

On the basis of small-molecule drugs, macromolecular drugs and cell therapy (e.g., stem cells) have attracted much attention because of their multi-target effects and promising clinical results, particularly for complicated ophthalmic diseases. Previous research has shown that hyaluronanchitosan nanoparticles can be absorbed by the corneal and conjunctival epithelial cells and further assimilated, suggesting the potential of polysaccharide-based nanocarriers for gene therapy (de La Fuente et al., 2008a,b). Chaharband et al. synthesized chitosan-hyaluronic acid nanopolyplexes loaded with siRNA through an ionic gelation method, which could penetrate the vitreous and retina barriers. Intravitreal injection experiments demonstrated that the nanopolyplexes could reach the posterior of rabbits and effectively reduce the size of laser-induced choroidal neovascularization (Chaharband et al., 2020). Biological macromolecules such as peptides can also be used to form polysaccharide-based hybrid nanocarriers (Qian et al., 2019; Lu et al., 2020). Silva et al. developed chitosanhyaluronic acid nanoparticles loaded with erythropoietin. In vitro permeation experiments showed rapid penetration into porcine conjunctiva followed by the sclera and cornea, with no cellular toxicity (Silva et al., 2020). Considering the therapeutic potential of stem cells for ophthalmological diseases, the development of polysaccharide-based cell-loading nanocarriers for further exploration is encouraging, especially for retinal and corneal stem cells.

Novel structural types of polysaccharide-based nanomaterials such as core-shell, hollow, and multi-layer structures have shown excellent drug delivery properties in terms of stability, drug-loading capacity, sustained release, corneal permeability, multi-stimuli sensitivity, and ocular bioavailability (Liu et al., 2018; Nie et al., 2019; Tan et al., 2019; Wei et al., 2020). Machado et al. synthesized a brimonidine-containing polymer$\beta$-cyclodextrin membrane with graphene oxide nanosheets and poly- $\beta$-aminoester intercalation. The drug release experiment suggested an association between the drug release kinetics and the number of graphene oxide nanosheet layers, making it easy to implement time-controlled drug release in the ocular system (Machado et al., 2019). Luo et al. prepared dual-functional nanoparticles by modifying chitosan and ZM241385 onto the surfaces of hollow ceria nanoparticles loaded with pilocarpine. The hollow structure significantly improved the drug retention. In addition, chitosan and ZM241385 were able to penetrate the cornea, while ceria elicited antioxidant and anti-inflammatory functions. These nanoparticles exhibited a 42 -fold longer period of lowering the intraocular pressure compared with that of commercial eye drops (Luo et al., 2020). Jiang et al. prepared a core-shell structured polysaccharide-based nanocarrier with a polycaprolactone shell and a chitosan core through a two-step emulsion method. The chitosan core was filled with bevacizumab via electrostatic interactions. These core-shell particles had a significantly improved capacity for long-term release of up to 3 months and possess good prospects for anti-VEGF therapeutics in clinical practice (Jiang et al., 2020).

In the synthesis of polysaccharide-based nanomaterials, some reagents may cause unpredictable toxicity toward normal tissues because of the limited available toxicological information compared with numerous synthetic compounds. Therefore, synthetic approaches based on green chemistry, such as self-assembly via hydrogen bonding, hydrophilic/hydrophobic interactions, Van der Waals forces, and electrostatic interactions, have attracted increasing interest (Han et al., 2020; Jin et al., 2020). Alqurshi et al. synthesized nanoparticles for delivering prednisolone acetate based on chitosan and sodium deoxycholate as a counterion through self-assembly. These nanocarriers 
performed better in terms of drug release behavior and antiinflammatory effects in the guinea pig model compared with micronized gel (Alqurshi et al., 2019). Several studies have attempted to modify polysaccharides simply to obtain various non-covalent interactions (Dubashynskaya N. V. et al., 2020; Liu C.H. et al., 2020).

\section{BEHAVIOR OF POLYSACCHARIDE-BASED NANOMATERIALS IN OCULAR TISSUE}

Before further clinical trials in patients with polysaccharide-based nanomaterials, their biological behavior and potential influence on the ocular system must be determined. Although emerging studies have emphasized the biocompatibility and biosafety of polysaccharides, a few have reported concerns regarding worsening the ocular pathology, including slightly elevated inflammatory factors and decreased densities of cones and rods (Yang et al., 2008; Jiang et al., 2018). In particular, as the size of materials decreases to the nanoscale, whether the corresponding changes in physical and chemical properties will have toxic effects should be further studied based on structural nanotoxicology (Zielińska et al., 2020). There have been several studies focusing on the biosafety of polysaccharide-based nanocarriers for ocular compartments; however, data on long-term observations and drug formulation are still lacking (De Campos et al., 2004; De Salamanca et al., 2006; Prow et al., 2008; Lai et al., 2010; Zorzi et al., 2011; Lai, 2012; Ogunjimi et al., 2017). Furthermore, the biodegradation of polysaccharide-based nanocarriers in the ocular system remains far from satisfactory, despite the fact that polysaccharides easily degrade in vivo (Etienne et al., 2005; Nguyen et al., 2019). Lai et al. detected polysaccharide degradation from drug carriers in lysozyme-containing buffers, a key metabolic regulator also present in the aqueous humor of the eyes, and suggested the possible enzymatic degradation of polysaccharide-based nanocarriers post-injection (Lai and Luo, 2017). However, at the nanoscale, some specific questions remain unclear, including where and how the polysaccharide-based nanocarrier is decomposed, metabolized, and excreted; what metabolites are produced by their degradation; and the possible impacts on the physiological function of the eye, particularly for hybrid nanomaterials. Emerging evidence has demonstrated

\section{REFERENCES}

Abdallah, M. M., Fernández, N., Matias, A. A., and Bronze, M. D. R. (2020). Hyaluronic acid and Chondroitin sulfate from marine and terrestrial sources: extraction and purification methods. Carbohydr. Polym. 243:116441. doi: 10. 1016/j.carbpol.2020.116441

Alaei, M., Atapour, M., and Labbaf, S. (2020). Electrophoretic deposition of chitosan-bioactive glass nanocomposite coatings on AZ91 $\mathrm{Mg}$ alloy for biomedical applications. Prog. Organ. Coatings 147:105803. doi: 10.1016/j. porgcoat.2020.105803

Alizadeh-Sani, M., Tavassoli, M., McClements, D. J., and Hamishehkar, H. (2021). Multifunctional halochromic packaging materials: saffron petal anthocyanin loaded-chitosan nanofiber/methyl cellulose matrices. Food Hydrocolloids 111:106237. doi: 10.1016/j.foodhyd.2020.106237 the biocompatibility and biosafety of polysaccharide-based nanocarriers based on material design, but there is a lack of effectiveness in real scenarios. In terms of the effect of controlled drug release in time-and-space evaluation, certain studies on polysaccharide-based nanocarriers, especially in the context of the physiological environment, remain controversial due to their differing animal models and research methodologies. In addition, as a sensory organ, visual function significantly affects patient compliance. Therefore, the influence of drug-delivery systems on visual quality should be considered. Nanoparticles are prone to aggregating because of their low surface energy, and as the size of aggregates increases, optometric diseases will emerge, such as vitreous opacity (Sultana et al., 2020). It is undeniable that the evaluation of visual quality is difficult, considering the physiological differences between human and animal models. Consequently, multi-disciplinary cooperation including ophthalmology, optometry, chemistry, materials science, and zoology should be encouraged. For instance, the development of biomimetic eye models based on engineering perspectives is expected to improve our understanding of the biological behavior of drug carriers (Bennet et al., 2020; Peng et al., 2020).

\section{DATA AVAILABILITY STATEMENT}

The original contributions presented in the study are included in the article/supplementary material, further inquiries can be directed to the corresponding author/s.

\section{AUTHOR CONTRIBUTIONS}

YF and HY devised the conception of the study. HY, WW, and XL wrote the manuscript. All authors contributed to the article and approved the submitted version.

\section{FUNDING}

This study was supported by a grant from the National Natural Science Foundation of China (No. 81700799) and Clinic Medicine Plus X-Young Scholars project, Peking University (The Fundamental research funds for the Central Universities).

Alqurshi, A., Hanafy, A. F., Abdalla, A. M., Guda, T. K., Gabr, K. E., and Royall, P. G. (2019). Ocular anti-inflammatory activity of prednisolone acetate loaded chitosan-deoxycholate self-assembled nanoparticles. Int. J. Nanomedicine 14, 3679-3689. doi: 10.2147/IJN.S195892

Anpilova, A. Y., Mastalygina, E. E., Khrameeva, N. P., and Popov, A. A. (2020). Methods for cellulose modification in the development of polymeric composite materials (Review). Russian J. Phys. Chem. B 14, 176-182. doi: 10.1134/ S1990793120010029

Asghar, M. A., Yousuf, R. I., Shoaib, M. H., and Asghar, M. A. (2020). Antibacterial, anticoagulant and cytotoxic evaluation of biocompatible nanocomposite of chitosan loaded green synthesized bioinspired silver nanoparticles. Int. J. Biol. Macromol. 160, 934-943. doi: 10.1016/j.ijbiomac.2020.05.197

Bazile, D. V. (2014). Nanotechnologies in drug delivery - An industrial perspective. J. Drug Deliv. Sci. Technol. 24, 12-21. doi: 10.1016/S1773-2247(14)50002-0 
Bennet, D., Estlack, Z., and Kim, J. (2020). “A microengineered human corneaon-a-chip for evaluating mass transport of ocular drugs," in Proceedings of the Chemical and Biological Microsystems Society (Lubbock, TX: Texas Tech University), 1102-1103.

Bennett, N. H., Chinnery, H. R., Downie, L. E., Hill, L. J., and Grover, L. M. (2020). Material, immunological, and practical perspectives on eye drop formulation. Adv. Funct. Mater. 30:1908476. doi: 10.1002/adfm.201908476

Bernd, A. S., Aihara, M., Lindsey, J. D., and Weinreb, R. N. (2004). Influence of molecular weight on intracameral dextran movement to the posterior segment of the mouse eye. Investig. Ophthalmol. Vis. Sci. 45, 480-484. doi: 10.1167/iovs. 03-0462

Bian, J., Peng, F., Peng, X.-P., Xu, F., Sun, R.-C., and Kennedy, J. F. (2012). Isolation of hemicelluloses from sugarcane bagasse at different temperatures: structure and properties. Carbohydr. Polym. 88, 638-645. doi: 10.1016/j.carbpol.2012.01. 010

Bian, J., Peng, F., Xu, F., Sun, R. C., and Kennedy, J. F. (2010). Fractional isolation and structural characterization of hemicelluloses from Caragana korshinskii. Carbohydr. Polym. 80, 753-760. doi: 10.1016/j.carbpol.2009.12.023

Cao, Y. X., Li, K., Qin, X. M., Jiao, S. M., Du, Y. G., and Li, X. R. (2019). Research approach on quality control of Astragalus polysaccharides based on polysaccharide receptor theory. Chinese Traditional Herb. Drugs 50, 2201-2209. doi: 10.7501/j.issn.0253-2670.2019.09.028

Chaharband, F., Daftarian, N., Kanavi, M. R., Varshochian, R., Hajiramezanali, M., Norouzi, P., et al. (2020). Trimethyl chitosan-hyaluronic acid nano-polyplexes for intravitreal VEGFR-2 siRNA delivery: formulation and in vivo efficacy evaluation. Nanomed. Nanotechnol. Biol. Med. 26:102181. doi: 10.1016/j.nano. 2020.102181

Chandel, L., Sharma, R., and Rana, V. (2019). Exploring the protective potential of carboxymethyl Terminalia catappa polysaccharide on blue light light-emitting diode induced corneal damage. Recent Patents Drug Deliv. Formul. 13, 310-322. doi: 10.2174/1872211314666191218110440

Chang, A. K. T., Frias, R. R., Alvarez, L. V., Bigol, U. G., and Guzman, J. P. M. D. (2019). Comparative antibacterial activity of commercial chitosan and chitosan extracted from Auricularia sp. Biocatal. Agric. Biotechnol. 17, 189-195. doi: 10.1016/j.bcab.2018.11.016

Chao, Y. C., Merritt, M., Schaefferkoetter, D., and Evans, T. G. (2020). Highthroughput quantification of protein structural change reveals potential mechanisms of temperature adaptation in Mytilus mussels. BMC Evol. Biol. 20:28. doi: 10.1186/s12862-020-1593-y

Chen, B., Zhou, K., Wang, Y., Xie, Y., Wang, Z., Li, P., et al. (2020). Insight into the mechanism of textural deterioration of myofibrillar protein gels at high temperature conditions. Food Chem. 330:127186. doi: 10.1016/j.foodchem. 2020.127186

Chen, S., Jia, H., Cui, X., Zhang, Y., Wen, Y., Ding, Y., et al. (2020). Characterization of stimuli-responsive and cross-linked nanohydrogels for applications in ophthalmiatrics therapy. Appl. Nanoscience 10, 3465-3475. doi: 10.1007/s13204-020-01450-7

Chien, K. J., Horng, C. T., Huang, Y. S., Hsieh, Y. H., Wang, C. J., Yang, J. S., et al. (2018). Effects of Lycium barbarum (goji berry) on dry eye disease in rats. Mol. Med. Rep. 17, 809-818. doi: 10.3892/mmr.2017.7947

De Campos, A. M., Diebold, Y., Carvalho, E. L. S., Sánchez, A., and Alonso, M. J. (2004). Chitosan nanoparticles as new ocular drug delivery systems: in vitro stability, in vivo fate, and cellular toxicity. Pharm. Res. 21, 803-810. doi: 10.1023/B:PHAM.0000026432.75781.cb

de La Fuente, M., Seijo, B., and Alonso, M. J. (2008a). Bioadhesive hyaluronanchitosan nanoparticles can transport genes across the ocular mucosa and transfect ocular tissue. Gene Therapy 15, 668-676. doi: 10.1038/gt.2008.16

de La Fuente, M., Seijo, B., and Alonso, M. J. (2008b). Novel hyaluronic acidchitosan nanoparticles for ocular gene therapy. Investig. Ophthalmol. Vis. Sci. 49, 2016-2024. doi: 10.1167/iovs.07-1077

De Oliveira Fulgêncio, G., Viana, F. A. B., Ribeiro, R. R., Yoshida, M. I., Faraco, A. G., and Da Silva Cunha-Júnior, A. (2012). New mucoadhesive chitosan film for ophthalmic drug delivery of timolol maleate: in vivo evaluation. J. Ocul. Pharmacol. Ther. 28, 350-358. doi: 10.1089/jop.2011.0174

De Salamanca, A. E., Diebold, Y., Calonge, M., García-Vazquez, C., Callejo, S., Vila, A., et al. (2006). Chitosan nanoparticles as a potential drug delivery system for the ocular surface: toxicity, uptake mechanism and in vivo tolerance. Investig. Ophthalmol. Vis. Sci. 47, 1416-1425. doi: 10.1167/iovs.05-0495
Dincă, V., Mocanu, A., Isopencu, G., Busuioc, C., Brajnicov, S., Vlad, A., et al. (2020). Biocompatible pure $\mathrm{ZnO}$ nanoparticles-3D bacterial cellulose biointerfaces with antibacterial properties. Arab. J. Chem. 13, 3521-3533. doi: 10.1016/j.arabjc.2018.12.003

Dubashynskaya, N., Poshina, D., Raik, S., Urtti, A., and Skorik, Y. A. (2020). Polysaccharides in ocular drug delivery. Pharmaceutics 12:22. doi: 10.3390/ pharmaceutics 12010022

Dubashynskaya, N. V., Golovkin, A. S., Kudryavtsev, I. V., Prikhodko, S. S., Trulioff, A. S., Bokatyi, A. N., et al. (2020). Mucoadhesive cholesterol-chitosan self-assembled particles for topical ocular delivery of dexamethasone. Int. J. Biol. Macromol. 158, 811-818. doi: 10.1016/j.ijbiomac.2020. 04.251

Etienne, O., Schneider, A., Taddei, C., Richert, L., Schaaf, P., Voegel, J. C., et al. (2005). Degradability of polysaccharides multilayer films in the oral environment: an in vitro and in vivo study. Biomacromolecules 6, 726-733. doi: 10.1021/bm049425u

Fan, X. M., Yu, H. Y., Wang, D. C., Yao, J., Lin, H., Tang, C. X., et al. (2019). Designing highly luminescent cellulose nanocrystals with modulated morphology for multifunctional bioimaging materials. ACS Appl. Mater. Interf. 11, 48192-48201. doi: 10.1021/acsami.9b13687

Felice, B., Prabhakaran, M. P., Rodríguez, A. P., and Ramakrishna, S. (2014). Drug delivery vehicles on a nano-engineering perspective. Mater. Sci. Eng. C 41, 178-195. doi: 10.1016/j.msec.2014.04.049

Ferreira, A. L., Charlie-Silva, I., Favero, G. C., Silva de Melo, N. F., Fraceto, L. F., Júnior, J. D. C., et al. (2020). Chitosan-coated zein nanoparticles containing eugenol potentiates anesthesia in Nile tilapia. Aquaculture 529:735659. doi: 10.1016/j.aquaculture.2020.735659

Flaxman, S. R., Bourne, R. R. A., Resnikoff, S., Ackland, P., Braithwaite, T., Cicinelli, M. V., et al. (2017). Global causes of blindness and distance vision impairment 1990-2020: a systematic review and metaanalysis. Lancet Glob. Health 5, e1221-e1234. doi: 10.1016/S2214-109X(17) 30393-5

Furlani, F., Sacco, P., Decleva, E., Menegazzi, R., Donati, I., Paoletti, S., et al. (2019). Chitosan acetylation degree influences the physical properties of polysaccharide nanoparticles: implication for the innate immune cells response. ACS Appl. Mater. Interfaces 11, 9794-9803. doi: 10.1021/acsami.8b21791

Garcia-Vaquero, M., O’Doherty, J. V., Tiwari, B. K., Sweeney, T., and Rajauria, G. (2019). Enhancing the extraction of polysaccharides and antioxidants from macroalgae using sequential hydrothermal-assisted extraction followed by ultrasound and thermal technologies. Mar. Drugs 17:457. doi: 10.3390/ md17080457

George, B., and Suchithra, T. V. (2019). Plant-derived bioadhesives for wound dressing and drug delivery system. Fitoterapia 137:104241. doi: 10.1016/j.fitote. 2019.104241

Hammi, N., El Hankari, S., Katir, N., Marcotte, N., Draoui, K., Royer, S., et al. (2020). Polysaccharide templated biomimetic growth of hierarchically porous metal-organic frameworks. Microporous Mesoporous Mater. 306:110429. doi: 10.1016/j.micromeso.2020.110429

Han, M., Liu, Y., Zhang, F., Sun, D., and Jiang, J. (2020). Effect of galactose sidechain on the self-assembly of xyloglucan macromolecule. Carbohydr. Polym. 246:116577. doi: 10.1016/j.carbpol.2020.116577

Irimia, T., Ghica, M. V., Popa, L., Anuta, V., Arsene, A. L., and Dinu-Pîrvu, C. E. (2018). Strategies for improving ocular drug bioavailability and cornealwound healing with chitosan-based delivery systems. Polymers 10:1221. doi: 10.3390/ polym 10111221

Jian, H. J., Wu, R. S., Lin, T. Y., Li, Y. J., Lin, H. J., Harroun, S. G., et al. (2017). Super-cationic carbon quantum dots synthesized from spermidine as an eye drop formulation for topical treatment of bacterial keratitis. ACS Nano 11, 6703-6716. doi: 10.1021/acsnano.7b01023

Jiang, P., Jacobs, K. M., Ohr, M. P., and Swindle-Reilly, K. E. (2020). Chitosan-polycaprolactone core-shell microparticles for sustained delivery of bevacizumab. Mol. Pharm. 17, 2570-2584. doi: 10.1021/acs.molpharmaceut. $0 \mathrm{c} 00260$

Jiang, X., Peng, Y., Yang, C., Liu, W., and Han, B. (2018). The feasibility study of an in situ marine polysaccharide-based hydrogel as the vitreous substitute. J. Biomed. Mater. Res. Pt A 106, 1997-2006. doi: 10.1002/jbm.a.36403

Jin, Y., Zhou, Q., Li, Z., Yang, Z., and Fan, H. J. S. (2020). Calcium-cross linked polysaccharide microcapsules for controlled release and antimicrobial 
applications. Coll. Surf. A Physicochem. Eng. Aspects 600:125025. doi: 10.1016/j. colsurfa.2020.125025

Joz Majidi, H., Babaei, A., Arab Bafrani, Z., Shahrampour, D., Zabihi, E., and Jafari, S. M. (2019). Investigating the best strategy to diminish the toxicity and enhance the antibacterial activity of graphene oxide by chitosan addition. Carbohydr. Polym. 225:115220. doi: 10.1016/j.carbpol.2019.115220

Karava, A., Lazaridou, M., Nanaki, S., Michailidou, G., Christodoulou, E., Kostoglou, M., et al. (2020). Chitosan derivatives with mucoadhesive and antimicrobial properties for simultaneous nanoencapsulation and extended ocular release formulations of dexamethasone and chloramphenicol drugs. Pharmaceutics 12, 1-29. doi: 10.3390/pharmaceutics12060594

Lai, J. Y. (2012). Biocompatibility of genipin and glutaraldehyde cross-linked chitosan materials in the anterior chamber of the eye. Int. J. Mol. Sci. 13, 10970-10985. doi: 10.3390/ijms130910970

Lai, J. Y., and Luo, L. J. (2017). Chitosan-g-poly(N-isopropylacrylamide) copolymers as delivery carriers for intracameral pilocarpine administration. Eur. J. Pharm. Biopharm. 113, 140-148. doi: 10.1016/j.ejpb.2016.11.038

Lai, J. Y., Luo, L. J., and Nguyen, D. D. (2020). Multifunctional glutathionedependent hydrogel eye drops with enhanced drug bioavailability for glaucoma therapy. Chem. Eng. J. 402:126190. doi: 10.1016/j.cej.2020.126190

Lai, J. Y., Ma, D. H. K., Cheng, H. Y., Sun, C. C., Huang, S. J., Li, Y. T., et al. (2010). Ocular biocompatibility of carbodiimide cross-linked hyaluronic acid hydrogels for cell sheet delivery carriers. J. Biomater. Sci. Polym. Ed. 21, 359-376. doi: $10.1163 / 156856209 X 416980$

Lakhani, P., Patil, A., and Majumdar, S. (2018). Recent advances in topical nano drug-delivery systems for the anterior ocular segment. Ther. Deliv. 9, 137-153. doi: 10.4155/tde-2017-0088

Lakshmanan, Y., Wong, F. S. Y., Zuo, B., So, K. F., Bui, B. V., and Chan, H. H. L. (2019). Posttreatment intervention with Lycium barbarum polysaccharides is neuroprotective in a rat model of chronic ocular hypertension. Investig. Ophthalmol. Vis. Sci. 60, 4606-4618. doi: 10.1167/iovs.19-27886

Li, H., Cao, J., Wu, X., Deng, Y., Ning, N., Geng, C., et al. (2020). Multiple fingerprint profiling for quality evaluation of polysaccharides and related biological activity analysis of Chinese patent drugs: zishen yutai pills as a case study. J. Ethnopharmacol. 260:113045. doi: 10.1016/j.jep.2020.113045

Li, Y. J., Luo, L. J., Harroun, S. G., Wei, S. C., Unnikrishnan, B., Chang, H. T., et al. (2019). Synergistically dual-functional nano eye-drops for simultaneous anti-inflammatory and anti-oxidative treatment of dry eye disease. Nanoscale 11, 5580-5594. doi: 10.1039/c9nr00376b

Liu, C. H., Lee, G. W., Wu, W. C., and Wang, C. C. (2020). Encapsulating curcumin in ethylene diamine- $\beta$-cyclodextrin nanoparticle improves topical cornea delivery. Coll. Surf. B Biointerfaces 186:110726. doi: 10.1016/j.colsurfb. 2019.110726

Liu, L., Sha, X. Y., Wu, Y. N., Chen, M. T., and Zhong, J. X. (2020). Lycium barbarum polysaccharides protects retinal ganglion cells against oxidative stress injury. Neural Regeneration Res. 15, 1526-1531. doi: 10.4103/1673-5374.274349

Liu, D., Lian, Y., Fang, Q., Liu, L., Zhang, J., and Li, J. (2018). Hyaluronic-acidmodified lipid-polymer hybrid nanoparticles as an efficient ocular delivery platform for moxifloxacin hydrochloride. Int. J. Biol. Macromol. 116, 10261036. doi: 10.1016/j.ijbiomac.2018.05.113

Liu, D., Wu, Q., Chen, W., Lin, H., Zhu, Y., Liu, Y., et al. (2019). A novel FK506 loaded nanomicelles consisting of amino-terminated poly(ethylene glycol)-block-poly(D,L)-lactic acid and hydroxypropyl methylcellulose for ocular drug delivery. Int. J. Pharm. 562, 1-10. doi: 10.1016/j.ijpharm.2019. 03.022

Liu, Y., and Zhang, Y. (2019). Lycium barbarum polysaccharides alleviate hydrogen peroxide-induced injury by up-regulation of miR-4295 in human trabecular meshwork cells. Exp. Mol. Pathol. 106, 109-115. doi: 10.1016/j.yexmp.2018. 12.007

Lodhi, B. A., Hussain, M. A., Ashraf, M. U., Farid-Ul-Haq, M., Haseeb, M. T., and Tabassum, T. (2020). Acute toxicity of a polysaccharide-based hydrogel from seeds of ocimum basilicum. Cell. Chem. Technol. 54, 291-299. doi: 10.35812/ CELLULOSECHEMTECHNOL.2020.54.31

Lu, T. Y., Huang, W. C., Chen, Y., Baskaran, N., Yu, J., and Wei, Y. (2020). Effect of varied hair protein fractions on the gel properties of keratin/chitosan hydrogels for the use in tissue engineering. Coll. Surf. B Biointerfaces 195:111258. doi: 10.1016/j.colsurfb.2020.111258
Luo, L. J., Nguyen, D. D., and Lai, J. Y. (2020). Dually functional hollow ceria nanoparticle platform for intraocular drug delivery: a push beyond the lim'temperate of static and dynamic ocular barriers toward glaucoma therapy. Biomaterials 243:119961. doi: 10.1016/j.biomaterials.2020.119961

Lynch, C., Kondiah, P. P. D., Choonara, Y. E., du Toit, L. C., Ally, N., and Pillay, V. (2019). Advances in biodegradable nano-sized polymer-based ocular drug delivery. Polymers 11:1371. doi: 10.3390/polym11081371

Machado, M., Silva, G. A., Bitoque, D. B., Ferreira, J., Pinto, L. A., Morgado, J., et al. (2019). Self-assembled multilayer films for time-controlled ocular drug delivery. ACS Appl. Bio Mater. 2, 4173-4180. doi: 10.1021/acsabm.9b00417

Mirzaeei, S., Berenjian, K., and Khazaei, R. (2018). Preparation of the potential ocular inserts by electrospinning method to achieve the prolong release profile of triamcinolone acetonide. Adv. Pharm. Bull. 8, 21-27. doi: 10.15171/apb. 2018.003

Mozammil Hasnain, S. M., Hasnain, M. S., and Nayak, A. K. (2019). "Chapter 1 - Natural polysaccharides: sources and extraction methodologies," in Natural Polysaccharides in Drug Delivery and Biomedical Applications, eds M. S. Hasnain and A. K. Nayak (Cambridge, MA: Academic Press), 1-14.

Müller, S., Ehlken, C., Bauer-Steinhusen, U., Lechtenfeld, W., Hasanbasic, Z., Agostini, H., et al. (2017). Treatment of age-related neovascular macular degeneration: the patient's perspective. Graefes Arch. Clin. Exp. Ophthalmol. 255, 2237-2246. doi: 10.1007/s00417-017-3739-1

Nagarwal, R. C., Singh, P. N., Kant, S., Maiti, P., and Pandit, J. K. (2010). Chitosan coated PLA nanoparticles for ophthalmic delivery: characterization, in-vitro and in-vivo study in rabbit eye. J. Biomed. Nanotechnol. 6, 648-657. doi: 10. $1166 /$ jbn. 2010.1168

Nakagawa, M., Tanaka, M., and Miyata, T. (1997). Evaluation of collagen gel and hyaluronic acid as vitreous substitutes. Ophthalmic Res. 29, 409-420. doi: $10.1159 / 000268042$

Nguyen, N. T.-P., Nguyen, L. V.-H., Tran, N. M.-P., Nguyen, D. T., Nguyen, T. N.-T., Tran, H. A., et al. (2019). The effect of oxidation degree and volume ratio of components on properties and applications of in situ cross-linking hydrogels based on chitosan and hyaluronic acid. Mater. Sci. Eng. C 103:109670. doi: 10.1016/j.msec.2019.04.049

Nie, J., Pei, B., Wang, Z., and Hu, Q. (2019). Construction of ordered structure in polysaccharide hydrogel: a review. Carbohydr. Polym. 205, 225-235. doi: 10.1016/j.carbpol.2018.10.033

Nishikawa, S., and Tamai, M. (1996). Ultrastructure of hyaluronic acid and collagen in the human vitreous. Curr. Eye Res. 15, 37-43. doi: 10.3109/ 02713689609017609

Ogunjimi, A. T., Melo, S. M. G., Vargas-Rechia, C. G., Emery, F. S., and Lopez, R. F. V. (2017). Hydrophilic polymeric nanoparticles prepared from Delonix galactomannan with low cytotoxicity for ocular drug delivery. Carbohydr. Polym. 157, 1065-1075. doi: 10.1016/j.carbpol.2016.10.076

Pan, Y., Liu, J., Yang, K., Cai, P., and Xiao, H. (2021). Novel multi-responsive and sugarcane bagasse cellulose-based nanogels for controllable release of doxorubicin hydrochloride. Mater. Sci. Eng. C 118:111357. doi: 10.1016/j.msec. 2020.111357

Pathak, K. (2019). Marine bioadhesives: opportunities and challenges. Ther. Deliv. 10, 749-751. doi: 10.4155/tde-2019-0070

Peng, Z., Zhou, L., Wong, J. K. W., and Chan, Y. K. (2020). Eye-on-a-chip (EOC) models and their role in the future of ophthalmic drug discovery. Expert Rev. Ophthalmol. 15, 259-261. doi: 10.1080/17469899.2020.1788388

Prow, T. W., Bhutto, I., Kim, S. Y., Grebe, R., Merges, C., McLeod, D. S., et al. (2008). Ocular nanoparticle toxicity and transfection of the retina and retinal pigment epithelium. Nanomed. Nanotechnol. Biol. Med. 4, 340-349. doi: 10. 1016/j.nano.2008.06.003

Qamar, Z., Qizilbash, F. F., Iqubal, M. K., Ali, A., Narang, J. K., Ali, J., et al. (2019). Nano-based drug delivery system: recent strategies for the treatment of ocular disease and future perspective. Recent Patents Drug Deliv. Formulation 13, 246-254. doi: 10.2174/1872211314666191224115211

Qian, Q., Niu, S., Williams, G. R., Wu, J., Zhang, X., and Zhu, L. M. (2019). Peptide functionalized dual-responsive chitosan nanoparticles for controlled drug delivery to breast cancer cells. Colloids Surfaces A Physicochem. Eng. Aspects 564, 122-130. doi: 10.1016/j.colsurfa.2018.12.026

Santana, C. P., Mansur, A. A. P., Mansur, H. S., and Silva-Cunha, A. D. Jr. (2020). Bevacizumab-conjugated quantum dots: in vitro antiangiogenic potential and 
biosafety in rat retina. J. Ocular Pharmacol. Ther. 36, 467-483. doi: 10.1089/jop. 2019.0108

Servais, A. B., Kienzle, A., Valenzuela, C. D., Ysasi, A. B., Wagner, W. L., Tsuda, A., et al. (2018). Structural heteropolysaccharide adhesion to the glycocalyx of visceral mesothelium. Tissue Eng. Part A 24, 199-206. doi: 10.1089/ten.tea.2017. 0042

Shah, A., Ashames, A. A., Buabeid, M. A., and Murtaza, G. (2020). Synthesis, in vitro characterization and antibacterial efficacy of moxifloxacin-loaded chitosan-pullulan-silver-nanocomposite films. J. Drug Deliv. Sci. Technol. 55:101366. doi: 10.1016/j.jddst.2019.101366

Shi, Y., Xiong, Z., Lu, X., Yan, X., Cai, X., and Xue, W. (2016). Novel carboxymethyl chitosan-graphene oxide hybrid particles for drug delivery. J. Mater. Sci. Mater. Med. 27:169. doi: 10.1007/s10856-016-5774-6

Silva, B., Marto, J., Braz, B. S., Delgado, E., Almeida, A. J., and Gonçalves, L. (2020). New nanoparticles for topical ocular delivery of erythropoietin. Int. J. Pharm. 576:119020. doi: 10.1016/j.ijpharm.2020.119020

Soliman, O. Y., Alameh, M. G., De Cresenzo, G., Buschmann, M. D., and Lavertu, M. (2020). Efficiency of chitosan/hyaluronan-based mRNA delivery systems in vitro: influence of composition and structure. J. Pharm. Sci. 109, 1581-1593. doi: 10.1016/j.xphs.2019.12.020

Sultana, S., Alzahrani, N., Alzahrani, R., Alshamrani, W., Aloufi, W., Ali, A., et al. (2020). Stability issues and approaches to stabilised nanoparticles based drug delivery system. J. Drug Target. 28, 468-486. doi: 10.1080/1061186X.2020. 1722137

Sun, D., Turner, J., Jiang, N., Zhu, S., Zhang, L., Falzon, B. G., et al. (2020). Atmospheric pressure microplasma for antibacterial silver nanoparticle/chitosan nanocomposites with tailored properties. Composites Sci. Technol. 186:107911. doi: 10.1016/j.compscitech.2019.107911

Taheri, P., Jahanmardi, R., Koosha, M., and Abdi, S. (2020). Physical, mechanical and wound healing properties of chitosan/gelatin blend films containing tannic acid and/or bacterial nanocellulose. Int. J. Biol. Macromol. 154, 421-432. doi: 10.1016/j.ijbiomac.2020.03.114

Tan, C., Arshadi, M., Lee, M. C., Godec, M., Azizi, M., Yan, B., et al. (2019). A robust aqueous core-shell-shell coconut-like nanostructure for stimuliresponsive delivery of hydrophilic cargo. ACS Nano 13, 9016-9027. doi: 10. 1021/acsnano.9b03049

Thacker, M., Tseng, C. L., Chang, C. Y., Jakfar, S., Chen, H. Y., and Lin, F. H. (2020). Mucoadhesive Bletilla striata polysaccharide-based artificial tears to relieve symptoms and inflammation in rabbit with dry eyes syndrome. Polymers 12:1465. doi: 10.3390/polym12071465

Tiwari, S., and Bahadur, P. (2019). Modified hyaluronic acid based materials for biomedical applications. Int. J. Biol. Macromol. 121, 556-571. doi: 10.1016/j. ijbiomac.2018.10.049

Uccello-Barretta, G., Balzano, F., Vanni, L., and Sansò, M. (2013). Mucoadhesive properties of tamarind-seed polysaccharide/hyaluronic acid mixtures: a nuclear magnetic resonance spectroscopy investigation. Carbohydr. Polym. 91, 568572. doi: 10.1016/j.carbpol.2012.07.085

Wang, H., Zhao, X., Huang, Y., Liao, J., Liu, Y., and Pan, Y. (2020). Rapid quality control of medicine and food dual purpose plant polysaccharides by matrix assisted laser desorption/ionization mass spectrometry. Analyst 145, 2168-2175. doi: 10.1039/c9an02440a

Wang, L., Wan, Y., Xu, Q., and Lou, X. (2019a). Long-term functional stability of functional nucleic acid-gold nanoparticle conjugates with different secondary structures. Langmuir 35, 11791-11798. doi: 10.1021/acs.langmuir.9b01884

Wang, Y., Bai, F., Luo, Q., Wu, M., Song, G., Zhang, H., et al. (2019b). Lycium barbarum polysaccharides grafted with doxorubicin: an efficient $\mathrm{pH}$-responsive anticancer drug delivery system. Int. J. Biol. Macromol. 121, 964-970. doi: 10.1016/j.ijbiomac.2018.10.103

Wang, Y., Lan, C., Liao, X., Chen, D., Song, W., and Zhang, Q. (2019c). Polygonatum sibiricum polysaccharide potentially attenuates diabetic retinal injury in a diabetic rat model. J. Diabetes Investig. 10, 915-924. doi: 10.1111/ jdi. 12976
Wei, Y., Cai, Z., Wu, M., Guo, Y., Wang, P., Li, R., et al. (2020). Core-shell pea protein-carboxymethylated corn fiber gum composite nanoparticles as delivery vehicles for curcumin. Carbohydr. Polym. 240:116273. doi: 10.1016/j.carbpol. 2020.116273

Yang, D., So, K. F., and Lo, A. C. Y. (2017). Lycium barbarum polysaccharide extracts preserve retinal function and attenuate inner retinal neuronal damage in a mouse model of transient retinal ischaemia. Clin. Exp. Ophthalmol. 45, 717-729. doi: 10.1111/ceo.12950

Yang, H., Wang, R., Gu, Q., and Zhang, X. (2008). Feasibility study of chitosan as intravitreous tamponade material. Graefes Arch. Clin. Exp. Ophthalmol. 246, 1097-1105. doi: 10.1007/s00417-008-0813-8

Yang, M., So, K. F., Lo, A. C. Y., and Lam, W. C. (2020). The effect of lycium barbarum polysaccharides on pyroptosis-associated amyloid $\beta 1-40$ oligomersinduced adult retinal pigment epithelium 19 cell damage. Int. J. Mol. Sci. 21, 1-16. doi: 10.3390/ijms21134658

Yang, Y., Ji, J., Di, L., Li, J., Hu, L., Qiao, H., et al. (2020). Resource, chemical structure and activity of natural polysaccharides against alcoholic liver damages. Carbohydr. Polym. 241:116355. doi: 10.1016/j.carbpol.2020.116355

Yellanki, S. K., Anna, B., and Kishan, M. R. (2019). Preparation and in vivo evaluation of sodium alginate - poly (vinyl alcohol) electrospun nanofibers of forskolin for glaucoma treatment. Pakistan J. Pharm. Sci. 32, 669-674.

Yuan, Y., Xu, X., Jing, C., Zou, P., Zhang, C., and Li, Y. (2018). Microwave assisted hydrothermal extraction of polysaccharides from Ulva prolifera: functional properties and bioactivities. Carbohydr. Polym. 181, 902-910. doi: 10.1016/j. carbpol.2017.11.061

Zhan, Z., Song, Q., Zhou, Z., and Lu, C. (2019). Ultrastrong and conductive MXene/cellulose nanofiber films enhanced by hierarchical nano-architecture and interfacial interaction for flexible electromagnetic interference shielding. J. Mater. Chem. C 7, 9820-9829. doi: 10.1039/c9tc03309b

Zhao, P., Li, X., Wang, Y., Yan, L., Guo, L., Huang, L., et al. (2020). Characterisation and saccharide mapping of polysaccharides from four common Polygonatum spp. Carbohydr. Polym. 233:115836. doi: 10.1016/j.carbpol.2020. 115836

Zheng, Y., Wang, Z., Xu, L., Wang, L., and He, W. (2018). Promoting repair effects of chitosan/gelatin electrospinning membrane implantation on rabbit conjunctival injury. Zhonghua Shiyan Yanke Zazhi Chinese J. Exp. Ophthalmol. 36, 102-106. doi: 10.3760/cma.j.issn.2095-0160.2018.02.006

Zheng, Z., Bian, S., Li, Z., Zhang, Z., Liu, Y., Zhai, X., et al. (2020). Catechol modified quaternized chitosan enhanced wet adhesive and antibacterial properties of injectable thermo-sensitive hydrogel for wound healing. Carbohydr. Polym. 249:116826. doi: 10.1016/j.carbpol.2020.116826

Zielińska, A., Costa, B., Ferreira, M. V., Miguéis, D., Louros, J. M. S., Durazzo, A., et al. (2020). Nanotoxicology and nanosafety: safety-by-design and testing at a glance. Int. J. Environ. Res. Public Health 17, 1-22. doi: 10.3390/ijerph17134657

Zorzi, G. K., Párraga, J. E., Seijo, B., and Sánchez, A. (2011). Hybrid nanoparticle design based on cationized gelatin and the polyanions dextran sulfate and chondroitin sulfate for ocular gene therapy. Macromol. Biosci. 11, 905-913. doi: 10.1002/mabi.201100005

Żukiewicz-Sobczak, W., Sobczak, P., Zawiślak, K., Zagórski, J., Wojtyła-Buciora, P., and Wojtyła, A. (2015). Physical and chemical properties comparison of fungal and crustaceous chitosan. J. Health Inequ. 1, 7-14. doi: 10.5114/jhi.2015.56820

Conflict of Interest: The authors declare that the research was conducted in the absence of any commercial or financial relationships that could be construed as a potential conflict of interest.

Copyright (c) $2020 \mathrm{Yu}, \mathrm{Wu}$, Lin and Feng. This is an open-access article distributed under the terms of the Creative Commons Attribution License (CC BY). The use, distribution or reproduction in other forums is permitted, provided the original author(s) and the copyright owner(s) are credited and that the original publication in this journal is cited, in accordance with accepted academic practice. No use, distribution or reproduction is permitted which does not comply with these terms. 